\title{
Caracterización de los servicios de promoción de la salud y prevención de la enfermedad en cuatro instituciones de salud del municipio de Medellín, 2015*
}

\section{Characterization of Health Promotion and Disease Prevention Services in Four Health Institutions in the Municipality of Medellín, 2015}

\section{Caracterização dos serviços de promoção da saúde e prevenção de doença em quatro instituições de saúde do município de Medellín, 2015}

Fecha de recepción: 23-10-2016 Fecha de aceptación: 30-03-2017 Disponible en línea: 30-05-2017 doi:10.11144/Javeriana.rgps16-33.csps

Cómo citar este artículo:

Barrientos-Gómez JG, Ángel-Jiménez G, López-Herrera FE. Caracterización de los servicios de promoción de la salud y prevención de la enfermedad en cuatro instituciones de salud del municipio de Medellín, 2015. Rev Gerenc Polít Salud. 2017; 16 (33): 60-77. https://doi.org/10.11144/Javeriana.rgps16-33.csps

\section{Juan Guillermo Barrientos-Gómez ${ }^{* *}$ \\ Gloria Ángel-Jiménez ${ }^{* * *}$ \\ Francy Edith López-Herrera ${ }^{* * *}$}

Artículo de investigación. Este trabajo fue financiado por la Universidad Pontificia Bolivariana. Por favor, dirigir la correspondencia a: Campus Robledo, Calle 78B \# 72A-109, Medellín, Antioquia, Colombia. Correo electrónico: juan.barrientos@upb.edu.co

** Médico, magíster en Investigación Sociosanitaria de la Universidad de Castilla La Mancha, docente de la Facultad de Medicina de la Universidad Pontificia Bolivariana. Correo electrónico: juan.barrientos@upb.edu.co

*** Magíster en Desarrollo de la Universidad Pontificia Bolivariana, docente de la Facultad de Enfermería de la Universidad Pontificia Bolivariana, Medellín, Colombia. Correo electrónico: gloria.angel@upb.edu.co

$60 * * *$ Magíster en Salud Pública de la Universidad CES, docente de la Facultad de Enfermería de la Universidad Pontificia Bolivariana, Medellín, Colombia. Correo electrónico: francy.lopez@upb.edu.co 


\section{Resumen}

Estudio descriptivo que permitió caracterizar los programas y las actividades de promoción de la salud y prevención de la enfermedad en cuatro instituciones de salud de alta complejidad de Medellín. Por medio de un estudio descriptivo de serie de casos, se aplicaron dos instrumentos semiestructurados tipo encuesta donde se recolectó información sobre: estructura y funcionamiento, enfoque, públicos beneficiarios, metodologías y equipos de trabajo. Se encontró que las cuatro instituciones desarrollan actividades de este tipo, con un enfoque hacia la prevención terciaria congruente con su nivel de complejidad; están orientadas esencialmente al paciente y a las familias, y, a veces, a la comunidad; los empleados también se benefician desde salud ocupacional; son desarrolladas por profesionales de diferentes disciplinas; su funcionamiento depende generalmente de las direcciones científicas; no se identifica un área en particular que las gestione articuladamente. Se considera importante que las instituciones se declaren a favor de modelos de atención en salud integrales donde se trabaje de manera articulada las actividades de promoción y prevención con la atención clínica.

Palabras clave: promoción de la salud; prevención de enfermedades; prevención primaria; prevención secundaria; prevención terciaria; hospitales; servicios hospitalarios

\section{Abstract}

This is a descriptive study that allowed for the characterization of the health promotion and disease prevention programs and activities in four high-complexity health institutions in Medellín. By means of a descriptive study of a series of cases, two semi-structured survey instruments were applied through which information was collected on: structure and functioning, approach, beneficiary communities, methodologies, and work teams. We found that all four institutions develop activities of this type, with a focus on tertiary prevention consistent with their level of complexity; they are essentially oriented to the patient and their families, and, sometimes, to the community; employees also benefit from occupational health; the activities are developed by professionals from different disciplines; their operation depends in general terms on scientific management; and no particular area was identified to manage the activities articulately. We considered important that the institutions manifest to be in favor of integral health care models where the promotion and prevention activities are coordinated with clinical care.

Keywords: health promotion; disease prevention; primary prevention; secondary prevention; tertiary prevention; hospitals, hospital services

\section{Resumo}

Estudo descritivo que permitiu caracterizar os programas e atividades de promoção da saúde e prevenção de doença em quatro instituições de saúde de alta complexidade de Medellín. Por meio de estudo descritivo de série de casos, aplicaram-se dois instrumentos semiestruturados tipo inquérito onde foi coletada informação sobre: estrutura e funcionamento, enfoque, públicos beneficiários, metodologias e equipes de trabalho. Verificou-se que as quatro instituições desenvolvem atividades deste tipo, com enfoque para a prevenção terciária congruente com o seu nível de complexidade; são orientadas essencialmente ao paciente e as famílias, e, às vezes, à comunidade; os empregados também são beneficiados desde saúde ocupacional; são desenvolvidas por profissionais de diferentes disciplinas; o seu funcionamento depende geralmente das direções científicas; não se identifica uma área em particular que as gere articuladamente. Considera-se importante as instituições se declararem em favor de modelos de atenção em saúde integrais onde se trabalharem de maneira articulada as atividades de promoção e prevenção com a atenção clínica.

Palavras-chave: promoção da saúde; prevenção da doença; prevenção primária; prevenção secundária; prevenção terciária; hospitais; serviços hospitalares 


\section{Introducción}

La promoción de la salud, según la Carta de Ottawa, consiste en proporcionar a los pueblos los medios necesarios para mejorar su salud y ejercer un mayor control sobre ella. El concepto de prevención de la enfermedad, por su parte, se utiliza para designar las estrategias tendientes a reducir los factores de riesgo de enfermedades específicas, o bien para reforzar factores personales que disminuyan la susceptibilidad a sufrirlas. Ambas estrategias pretenden generar reflexión para la acción, concretada por medio de hábitos y estilos de vida que se espera sean adquiridos por las personas para que contribuyan al cuidado de su salud (1-2).

De acuerdo con lo planteado por Laevell y Clark en 1965, las intervenciones de promoción y prevención tienen como fin evitar el desarrollo de la historia natural y social de las enfermedades, por medio de intervenciones primarias, secundarias o terciarias (3). Se considera, además, que estas intervenciones son cada vez más necesarias, dada la transición epidemiológica a enfermedades crónicas no transmisibles (4) y el comportamiento de enfermedades infecciosas que se conservan en los países en vía de desarrollo, todas ellas ligadas de manera importante a hábitos inadecuados que favorecen su ocurrencia, lo que no significa que no existan otros riesgos o factores asociados a su ocurrencia.

Dada la importancia de las acciones de promoción de la salud y prevención de la enfermedad, se esperaría que estas fueran un pilar fundamental en el direccionamiento del sistema de salud colombiano; sin embargo, la situación actual muestra que la responsabilidad de su desarrollo recae, principalmente, en las empresas de aseguramiento en salud y secretarías de salud, las cuales no alcanzan a lograr lo esperado en este sentido, dado que centran su actividad en la solución de problemas prioritarios y dejan por fuera otras situaciones que pueden generar riesgos para la salud (5). De otro lado, los modelos de prestación de servicios, así como los de formación profesional, tienen un enfoque curativo, lo cual no posibilita el logro de los beneficios que pueden obtenerse mediante el desarrollo de acciones de promoción y prevención (6).

Con relación al desarrollo de acciones de promoción de la salud y prevención de la enfermedad en las entidades prestadoras de servicios de salud, también debe anotarse que estas, tradicionalmente, se ejecutan en los primeros niveles de atención. Las instituciones de mediana y alta complejidad no tienen un papel contundente en este campo, dado su modelo de atención curativo. De esta forma, se limita la realización de una atención integral en la prestación de servicios al paciente y su grupo familiar.

La situación expuesta evidencia que es necesario implementar estrategias diversas que permitan abordar el proceso salud-enfermedad de una forma integral, con la participación de todos los actores del sistema de salud y que busquen intervenir en los hábitos de la población, desde medidas de promoción de la salud y de modificación de factores de riesgo (7-8).

Se considera importante replantear el modelo de prestación de servicios asistenciales, no solo en las unidades de atención básica, sino también en los hospitales de mayor complejidad, de tal forma que incluyan medidas que fomenten la promoción y, adicionalmente, consideren acciones preventivas para abordar los riesgos más frecuentes que generan enfermedad. Dicho enfoque se ha desarrollado desde los años noventa, según los lineamientos de la Organización Mundial de la Salud (OMS), en los países europeos, bajo el concepto de hospitales promotores de la salud (9), y ha alcanzado modificaciones reales en los modelos de atención en salud. 
Para el diseño y la implementación de un modelo de prestación de servicios asistenciales que promueva la salud y prevenga las enfermedades, se ha considerado importante desarrollar investigaciones de diversa índole que generen conocimiento para el logro de este fin, dado que en el medio es baja la disponibilidad de información acerca del tema. Por este motivo, se planteó la presente investigación, la cual buscó caracterizar los programas y las actividades de promoción de la salud y prevención de la enfermedad que tenían algunas instituciones de salud de alta complejidad de Medellín.

\section{Materiales y métodos}

Con un enfoque cuantitativo, se realizó un estudio descriptivo tipo serie de casos, que caracterizó los diferentes programas y actividades de promoción de la salud y prevención de la enfermedad que se desarrollan en cuatro instituciones de salud de alta complejidad de la ciudad de Medellín, elegidas de forma intencional por su ubicación en la zona geográfica de referencia, además de su trayectoria en el medio.

Las variables estudiadas se agruparon en dos grupos. El primero, relacionado con las características institucionales, entre ellas: naturaleza de la institución, existencia de programas y actividades, su enfoque e instancia organizacional responsable. E1 segundo estuvo relacionado con las características de los programas o actividades propiamente dichos, tales como: persona responsable, formación, equipo que los desarrolla, población beneficiaria, dependencia operativa, descripción de las acciones desarrolladas, utilización de guías y mecanismos de seguimiento y contratación.

Así, para la recolección de la información se diseñaron dos tipos de instrumentos semiestructurados que contemplaron preguntas relacionadas con las variables de interés, los cuales se aplicaron mediante entrevistas a los responsables del proceso en cada institución y a los líderes de los programas y actividades identificados.

Los instrumentos se sometieron previamente a una prueba piloto para minimizar sesgos de información. Además, cada entrevista fue grabada y desarrollada por mínimo dos investigadores. Los datos obtenidos se confrontaron con información institucional para controlar sesgos de memoria.

El análisis de los resultados se centró en los siguientes componentes: estructura y funcionamiento; enfoque de los programas y las actividades; públicos beneficiarios; metodologías o estrategias educativas y equipos de trabajo. El análisis se orientó a identificar los elementos comunes o diferenciadores, los cuales se confrontaron a la luz de la teoría existente en los modelos de organización de servicios en instituciones de salud.

Desde el punto de vista ético, el estudio se clasificó como una investigación sin riesgo, según la Resolución 008430 de 1993, y teniendo en cuenta que no se indagó a los entrevistados por opiniones o experiencias personales, solo por lo que desde su rol de trabajadores pudieran conocer de los programas y las actividades que se desarrollan en la institución de salud donde laboran. Se obtuvo la aprobación previa del Comité de Investigación en Salud de la Universidad Pontificia Bolivariana, además de la autorización de cada una de las instituciones participantes. Se dio confidencialidad a la información de las fuentes y datos obtenidos.

\section{Resultados}

La descripción general de las características de las instituciones de salud estudiadas muestra que se trata de organizaciones de natura- 
leza privada, de alto nivel de complejidad, dos de las cuales contemplan servicios generales en una amplia gama de especialidades médicas y servicios especializados, orientados al binomio madre-hijo en la primera y a personas con problemas cardiovasculares en la segunda. Las cuatro instituciones consultadas constituyen un referente en el ámbito local, departamental y nacional.

A continuación, se describen, por institución, los resultados alusivos a los programas y las actividades de promoción de la salud y prevención de la enfermedad.

\section{Institución 1}

Esta institución cuenta con diversos programas y actividades enfocados en la promoción de la salud o prevención de la enfermedad, desarrollados por grupos interdisciplinarios. La población objeto es tanto el público externo (usuarios, familia, visitantes, comunidad en general) como el interno (personal administrativo y asistencial). Estos programas son liderados por diferentes instancias institucionales como la coordinación científica o médica y la coordinación de enfermería, comunicaciones y gestión humana, entre otras. No existe un grupo de base que se encargue del desarrollo de todos ellos, sino que cada uno está dirigido y organizado por diferentes áreas. Con respecto a la formación profesional de los responsables de su desarrollo, figuran, entre otros: médicos generales y especialistas, profesionales de enfermería, trabajadores sociales, psicólogos, comunicadores y auxiliares de enfermería. La asignación en horas de estos es variable, algunos tienen dedicación exclusiva, otros de medio tiempo, los demás participan en actividades puntuales según la necesidad o temática que se vaya a desarrollar; ejemplo de ello son los especialistas.

64 Los programas o actividades que se desarrollan tienen un enfoque orientado, principalmente, a la prevención terciaria y, en menor proporción, a la promoción de la salud y la prevención primaria y secundaria. Las personas afiliadas al régimen subsidiado, al contributivo o a regímenes especiales del Sistema General de Seguridad Social en Salud pueden acceder a los diferentes servicios que ofrece la institución.

Todos los programas o actividades que se desarrollan tienen una plataforma de gestión y están documentados. Su seguimiento y evaluación se hace a través de indicadores de resultados y encuestas de satisfacción.

Los programas y las actividades de prevención terciaria que se desarrollan en la institución están dirigidos a pacientes con una condición de salud-enfermedad de base —falla cardíaca, diabetes, anticoagulados, obesidad infantil- y tienen como objetivo contribuir a la rehabilitación de su situación. Un elemento transversal de estas acciones es la educación para la salud.

Los resultados también permiten identificar otro tipo de acciones orientadas no solo a pacientes, sino también a grupos poblacionales diversos, las cuales se centran en la prevención primaria y secundaria. Algunas de estas son: jornadas de prevención del cáncer de mama, fomento de la lactancia materna, talleres educativos de cocina saludable, campañas de detección temprana de glaucoma y ceguera por diabetes, entre otras actividades educativas abiertas a la comunidad.

Para empleados y sus familias, se realizan programas orientados al fortalecimiento de las relaciones y la administración del hogar. Se promueve la salud por medio del deporte, el arte y otros estilos de vida saludable; se celebran días especiales; se hacen orientaciones en la compra de vivienda; se les acompaña en su periodo de preparación para la jubilación; también se ofrece un programa interdisciplinario de prevención adicciones 
dirigido a empleados, usuarios y al público en general.

Los elementos de información, comunicación y educación se soportan desde el área de comunicaciones, la cual, adicionalmente, lidera algunas acciones de promoción de la salud dirigidas a público interno y externo, en diferentes temas como: cocina saludable, oftalmología, optometría, lactancia materna, entre otros, según programación anual. Lo medios utilizados para tal objetivo son impresos, uso de plataformas virtuales y programas radiales y televisivos.

\section{Institución 2}

Los resultados encontrados muestran que esta institución posee diversos programas o actividades que tienen como fin promover la salud o prevenir la enfermedad. Estos se adscriben a diferentes instancias organizacionales, aunque la más frecuente es la división médica. Todos los programas estudiados tienen un profesional de la salud que los lidera, quien está apoyado por un equipo asistencial compuesto por profesionales de diferentes áreas de la salud, de acuerdo con el perfil del programa; entre ellos, médicos, enfermeros, nutricionistas, psicólogos, trabajadores sociales y comunicadores.

Los programas y las actividades de promoción y prevención orientados a los empleados de la organización son dirigidos desde salud ocupacional, la cual depende de la Dirección de Gestión Humana, y son liderados por un médico especialista en salud ocupacional, cuya labor es apoyada por las siguientes personas: profesional y auxiliar de enfermería, psicólogo fisioterapeuta y practicante de educación física. Por su parte, el Programa de Información y Comunicación depende de la Gerencia y está liderado por un comunicador, quien se apoya de manera colaborativa en diferentes profesionales, de acuerdo con el objetivo educativo específico.

Con respecto a los beneficiarios de los programas o actividades identificados, se encontró que estos se dirigen al público institucional: pacientes, usuarios, familia, cuidadores, visitantes y empleados. Y algunos se orientan al público externo: estudiantes, trabajadores y personas de la comunidad en general.

Algunos de los programas y las actividades de atención se desarrollan bajo el esquema de clínicas especializadas y tienen un enfoque principal en prevención terciaria. Se desarrollan acciones de atención integral, seguimiento y control de complicaciones, así como acciones educativas individuales y grupales dirigidas a los pacientes y a sus familias. También realizan jornadas de salud en colegios, universidades y empresas con capacitaciones, esencialmente en diabetes, disfagia, hemofilia, tabaquismo y obesidad.

Los programas y las actividades de promoción de la salud y prevención de la enfermedad para los empleados son liderados por el área de salud ocupacional y se desarrollan en dos frentes, uno está orientado al fomento de la calidad de vida de los trabajadores y el otro a la identificación y el control de sus factores de riesgo. Para ello, la institución cuenta con los siguientes programas: Riesgo Cardiovascular, Actívate, Futuras Mamás, Conservación Visual y Vacunación No Ocupacional.

Para la comunidad en general, la institución cuenta con un programa extramural, el cual se desarrolla en un municipio previamente seleccionado. Las acciones se dirigen, principalmente, a líderes comunitarios y al personal de salud del hospital municipal. A escala poblacional, también se desarrollan actividades orientadas a la prevención y la detección precoz del cáncer; además, apoyan 
la gestión para la atención, el tratamiento y la asistencia social de estos pacientes.

La gestión de los diferentes programas está enmarcada dentro del sistema de gestión de la calidad institucional, lo cual significa que están definidos con base en los lineamientos del sistema y que su planeación y seguimiento están debidamente documentadas.

Los elementos de información, comunicación y educación para la salud institucionales que se orientan a la promoción de la salud y la prevención de la enfermedad, se desarrollan con base en las características de la población que se atiende y cuentan con procesos estructurados en los siguientes temas: diabetes mellitus, cuidados paliativos, obesidad, manejo de ostomías, tabaquismo, trauma raquimedular, disfagia, polimedicación y anticoagulación. Utilizan diferentes medios de información para cumplir con su objetivo, como el canal de comunicación interno, programas radiales, redes sociales y carteleras. También cuentan con diferentes diseños litográficos como material de apoyo a los procesos de educación.

\section{Institución 3}

La institución cuenta con programas y actividades de promoción de la salud y prevención de la enfermedad en sus tres niveles, con énfasis en el terciario, dirigidas a la rehabilitación de pacientes con patología cardiovascular o pulmonar, coherente con su especialidad.

No se identifica una coordinación que integre los programas o actividades que se desarrollan en la institución. Cada una de las áreas asistenciales ejecuta directamente las actividades de forma independiente, en articulación con la Dirección Científica. Adicionalmente, la dinámica de estos se en66 cuentra integrada al área de piscología, la cual tiene un papel fundamental en el fomento de hábitos y estilos de vida saludables y, además, apoya las actividades de prevención terciaria, dirigidas a la rehabilitación de los pacientes inscritos en cada uno de los programas.

Los públicos de los programas y las actividades son diversos e incluyen pacientes, familiares o cuidadores, empleados de la institución, personal de empresas de la ciudad y población general. Los pacientes beneficiarios de estos programas casi siempre presentan una condición clínica compleja, por lo que requieren acompañamiento profesional que les permita, a ellos y a sus familias, reconocer su enfermedad, asumir los procesos de intervención, participar en las actividades de rehabilitación y adaptarse a las nuevas condiciones de vida. El abordaje de los pacientes y sus familias tiene un enfoque psicoeducativo liderado por el área de psicología, mencionada anteriormente.

Las actividades dirigidas al equipo humano colaborador de la institución son lideradas por el área de salud ocupacional. Desde el ingreso se identifican riesgos y se orienta al trabajador para que los intervenga desde los programas o actividades que la institución le ofrece, o bien desde los servicios de promoción o prevención que tiene la empresa promotora de salud (EPS) a la cual está inscrito. La institución cuenta con un plan de bienestar institucional, a través del cual se desarrollan actividades de promoción de la actividad física, vacunación, pausas activas, vigilancia de desórdenes músculo-esqueléticos y control de riesgos psicosociales. Adicionalmente, cuenta con una semana de la salud para los empleados, en la cual se desarrollan actividades diversas de promoción y prevención.

El desarrollo de muchas de las actividades mencionadas no es cubierto por las empresas aseguradoras de salud. Por tanto, se realizan como una acción de tipo misional de la institución que buscan dar un servicio integral en salud. 
La institución cuenta con un sistema de calidad que permite la garantía de la prestación de estos servicios, evidenciado por el apoyo documental, que incluye protocolos, guías y soportes de las atenciones prestadas y el seguimiento por medio de indicadores.

Los elementos de información, comunicación y educación para la salud que se identifican en esta institución se materializan en la actividad psicoeducativa, la cual se desarrolla de manera transversal a todos los programas de prevención terciaria. Además, dichos elementos se despliegan en el área de comunicaciones, la cual cuenta con una importante plataforma de medios que pone a disposición de los diferentes públicos beneficiarios, entre los cuales figuran: boletines físicos y virtuales, carteleras, folletos, portal web, Facebook, Youtube, canal interno de televisión, programa de televisión externo y una línea telefónica de consulta donde se responden preguntas a la población.

\section{Institución 4}

Los programas y las actividades de promoción y prevención identificados en esta institución son ejecutados por equipos que trabajan de forma independiente. De manera específica, la coordinación de servicios ambulatorios desarrolla varias actividades de esta índole. No obstante, la dirección asistencial de la institución es la instancia que los congrega

El enfoque principal de las actividades que se desarrollan es preventivo. Asimismo, se llevan cabo acciones orientadas al fomento de conductas saludables, como lo es la promoción de la lactancia materna. La educación para la salud constituye un elemento transversal de todas ellas.

Con respecto a los equipos que desarrollan los programas y las actividades, se encontró que los profesionales de enfermería son, en gran medida, los responsables de su funcionamiento, quienes cuentan con el apoyo de otros profesionales según las características o necesidades de los diferentes programas. Un profesional que realiza el servicio social obligatorio en el área de enfermería tiene una destinación específica a esta labor.

Los públicos beneficiarios de los programas y las actividades son diversos e incluyen tanto a los pacientes y sus familiares o cuidadores como al público en general. Se prestan servicios a todos los usuarios del sistema de seguridad social y regímenes excepcionales.

Algunos de los programas y las actividades orientados a los pacientes y sus familias son: programa IAMI —derivado de la política de Instituciones Amigas de la Mujer y de la Infancia-; Atención a Pacientes Quirúrgicos - con énfasis en cirugía de prótesis, bariátrica y piso pélvico-; Manejo de Heridas y Ostomías en Casa; Prevención de Úlceras por Presión y de Caídas; y Cuidados del Recién Nacido Sano o con Prematurez. Este último, apoyado por una fundación conformada por empleados que, adicionalmente, apoyan a las familias con subsidios de trasporte, alimentación e insumos para el cuidado del bebé.

Para el grupo humano de la institución, el área de salud ocupacional cuenta con actividades orientadas a la prevención de alteraciones propias del contexto clínico. además, tiene un programa de ausentismo que se orienta a la prevención de patologías osteomusculares y mentales. El programa de bienestar laboral institucional realiza actividades para el fomento de la calidad de vida en el trabajo y celebraciones para el reconocimiento del personal. De igual manera, se coordinan actividades con la administradora de riesgos laborales (ARL) como la campaña Cuídate que Yo te Cuido, jornadas de tamizajes cáncer de seno y próstata- $\mathrm{y}$ actividades de integración familiar. 
La institución desarrolla estas actividades en el marco del mejoramiento continuo de los procesos del sistema obligatorio de garantía de calidad, con el apoyo documental respectivo, haciendo seguimiento por indicadores de gestión.

Los elementos de información, comunicación y educación para la salud son orientados desde la consulta externa para los pacientes ambulatorios y desde el departamento de enfermería para los usuarios hospitalizados. Estas áreas apoyan el desarrollo de los diferentes programas de promoción y prevención de la organización. El material para las diferentes actividades educativas es elaborado por el área de comunicación, la cual tiene a cargo acciones de información y comunicación que desarrolla a través de varias estrategias como los volantes para empleados con temas de interés, así como las carteleras donde se divulga información para profesionales y el público en general, en temas alusivos al cuidado de la salud y eventos especiales.

Una vez descritos los resultados de cada una de las instituciones participantes, los investigadores decidieron presentar los diferentes programas y las actividades que se encontraron en cada una de ella, con el propósito de visibilizar la labor que desarrollan estas instituciones en lo relacionado con la prevención de la enfermedad en su tres niveles: primaria con acciones educativas y secundaria con actividades de detección precoz y estrategias que acompañan a pacientes y familias para que recuperen su salud o se involucren activamente en su cuidado desde la prevención terciaria (10). En la tabla 1 se presentan los programas y las actividades, según el nivel de prevención y la institución que los desarrolla.

TABla 1. Programas Y ACTIVIDADES DE PREVENCIÓN Y PROMOCIÓN POR INSTITUCIONES

\begin{tabular}{|c|c|c|c|c|}
\hline Actividad & Institución 1 & Institución 2 & Institución 3 & Institución 4 \\
\hline $\begin{array}{c}\text { Prevención } \\
\text { terciaria }\end{array}$ & $\begin{array}{l}\text { Programa para } \\
\text { pacientes con } \\
\text { falla cardiaca } \\
\text { Clínica de } \\
\text { diabetes } \\
\text { Programa de } \\
\text { anticoagulados } \\
\text { Atención a } \\
\text { pacientes con } \\
\text { obesidad }\end{array}$ & $\begin{array}{l}\text { Diabetes } \\
\text { Programa para pacientes } \\
\text { con Disfagia } \\
\text { Clínica de Hemofilia } \\
\text { Clínica de Tabaquismo } \\
\text { Atención de la } \\
\text { Obesidad-Cirugía Ba- } \\
\text { riátrica } \\
\text { Programa de Cuidados } \\
\text { Paliativos } \\
\text { Programa de Ostomías } \\
\text { Atención de Pacientes } \\
\text { con Trauma Raquime- } \\
\text { dular } \\
\text { Programa para Pacientes } \\
\text { Polimedicados } \\
\text { Programa de } \\
\text { Anticoagulados }\end{array}$ & $\begin{array}{l}\text { Programa para } \\
\text { atención de } \\
\text { pacientes con falla } \\
\text { cardiaca } \\
\text { Rehabilitación } \\
\text { cardiovascular } \\
\text { Programa de tras- } \\
\text { plante de corazón y } \\
\text { pulmón } \\
\text { Programa para } \\
\text { pacientes } \\
\text { anticoagulados }\end{array}$ & $\begin{array}{l}\text { Programa de aten- } \\
\text { ción a pacientes } \\
\text { con artrosis de } \\
\text { cadera y rodilla: } \\
\text { cirugía de prótesis } \\
\text { Atención al pacien- } \\
\text { te con obesidad-ci- } \\
\text { rugía bariátrica } \\
\text { Clínica de pato- } \\
\text { logías del piso } \\
\text { pélvico } \\
\text { Clínica de heridas y } \\
\text { ostomías } \\
\text { Plan Camino a } \\
\text { Casa, dirigido a } \\
\text { familias con recién } \\
\text { nacidos con necesi- } \\
\text { dades especiales de } \\
\text { atención }\end{array}$ \\
\hline
\end{tabular}




\begin{tabular}{|c|c|c|c|c|}
\hline $\begin{array}{l}\text { Prevención } \\
\text { secundaria }\end{array}$ & $\begin{array}{l}\text { Tamizaje en } \\
\text { salud visual }\end{array}$ & $\begin{array}{l}\text { Tamizaje diabetes } \\
\text { Identificación de riesgo } \\
\text { cardiovascular } \\
\text { Tamizaje en salud visual } \\
\text { Programa de atención } \\
\text { complementaria a la } \\
\text { gestante }\end{array}$ & $\begin{array}{l}\text { Vigilancia de } \\
\text { desórdenes osteo- } \\
\text { musculares en el } \\
\text { empleado } \\
\text { Vigilancia de } \\
\text { riesgo psicosocial } \\
\text { al empleado } \\
\text { Programa de au- } \\
\text { sentismo laboral }\end{array}$ & $\begin{array}{l}\text { Tamizaje cáncer de } \\
\text { seno al empleado } \\
\text { Tamizaje de cáncer } \\
\text { de próstata al } \\
\text { empleado }\end{array}$ \\
\hline $\begin{array}{c}\text { Prevención } \\
\text { primaria }\end{array}$ & $\begin{array}{l}\text { Educación en } \\
\text { cáncer (mama) } \\
\text { Programa y } \\
\text { actividades en } \\
\text { el marco de } \\
\text { la política de } \\
\text { Instituciones } \\
\text { Amigas de la } \\
\text { Mujer y de } \\
\text { la Infancia: } \\
\text { lactancia } \\
\text { materna, pue- } \\
\text { ricultura }\end{array}$ & $\begin{array}{l}\text { Educación sobre hábitos } \\
\text { a pacientes en riesgo: } \\
\text { alimentación sana, pre- } \\
\text { vención del tabaquismo } \\
\text { y fomento de la actividad } \\
\text { física } \\
\text { Programa de vacunación } \\
\text { no ocupacional }\end{array}$ & $\begin{array}{l}\text { Programa de apoyo } \\
\text { psicoeducativo } \\
\text { Educación sobre } \\
\text { hábitos saludables } \\
\text { a pacientes en } \\
\text { riesgo } \\
\text { Programa de vacu- } \\
\text { nación } \\
\text { Programa de } \\
\text { actividad física } \\
\text { pausas activas en el } \\
\text { trabajo }\end{array}$ & $\begin{array}{l}\text { Programa y } \\
\text { actividades en el } \\
\text { marco de la política } \\
\text { de Instituciones } \\
\text { Amigas de la Mujer } \\
\text { y de la Infancia: } \\
\text { lactancia materna, } \\
\text { puericultura }\end{array}$ \\
\hline $\begin{array}{l}\text { Promoción } \\
\text { de la salud }\end{array}$ & $\begin{array}{l}\text { Promoción } \\
\text { de hábitos } \\
\text { saludables } \\
\text { Jornada sobre } \\
\text { lactancia ma- } \\
\text { terna } \\
\text { Actividades } \\
\text { culturales y de- } \\
\text { portivas para } \\
\text { los empleados } \\
\text { y sus familias }\end{array}$ & $\begin{array}{l}\text { Promoción de hábitos } \\
\text { saludables a empleados, } \\
\text { estudiantes de colegios, } \\
\text { universidades y público } \\
\text { en general }\end{array}$ & $\begin{array}{l}\text { Promoción de hábi- } \\
\text { tos saludables para } \\
\text { públicos diversos }\end{array}$ & $\begin{array}{l}\text { Promoción de hábi- } \\
\text { tos saludables } \\
\text { Actividades orien- } \\
\text { tadas al fomento de } \\
\text { la calidad de vida } \\
\text { en el trabajo: estí- } \\
\text { mulos integración } \\
\text { familiar y campa- } \\
\text { ñas de autocuidado }\end{array}$ \\
\hline
\end{tabular}

\section{Discusión}

Este apartado presentará el producto de la discusión realizada alrededor de los aspectos más relevantes de los programas y las actividades de promoción de la salud y prevención de la enfermedad de cada institución, los cuales tienen que ver con: estructura y funcionamiento, enfoque principal, públicos beneficiarios, equipos de trabajo y metodologías o estrategias educativas empleadas.

\section{Estructura y funcionamiento}

Un primer hallazgo que amerita mencionarse tiene que ver con la estructura organizacional que soporta los programas y las actividades de promoción de la salud y prevención de la enfermedad. Al respecto, se encontró que la mayoría de estos son liderados por la dirección de servicios asistenciales o quien haga sus veces en las instituciones participantes. Adicionalmente, su desarrollo está, por lo general, adscrito a servicios de carácter ambulatorio y tiene una dinámica de trabajo independiente, con algunas actividades en común, relacionadas principalmente con educación y comunicación en salud. Este hallazgo difiere de lo contemplado en el Decreto 1876 de 1994 (11), el cual ubica la atención en promoción y prevención en el área ambulatoria de atención al usuario, lo 
que puede deberse a la organización interna que cada institución da a sus servicios de acuerdo con su misión, intereses, recursos y capacidades, entre otros.

Además del área asistencial, la de comunicaciones y la de salud ocupacional trabajan con fuerte interrelación para el desarrollo de los diferentes programas y las actividades de promoción y prevención. Asimismo, coordinan acciones con los servicios de internación, lo que es fundamental para lograr el propósito de la atención integral de los servicios médico-asistenciales (12).

Los lineamientos definidos por el Ministerio de Salud colombiano, a través de la Resolución 00412 del 2000, contemplan las guías de atención de los programas de protección específica, detección temprana y atención a las enfermedades de interés en salud pública que deben desarrollarse en las instituciones de salud. No obstante, en las participantes estos programas no están configurados de esta forma, dado su nivel de complejidad y las condiciones clínicas de sus pacientes (13).

Los diferentes programas y las actividades cuentan con evaluaciones de resultados de su gestión, lo cual se realiza por medio de programas de vigilancia para el control de enfermedades y gestión de la información clínica, con el fin de reducir riesgos, mejorar la utilización de los recursos y proporcionar la mejor atención posible (14).

Es de anotar que los programas y las actividades de promoción y prevención deben estar enmarcados en el modelo de atención de las instituciones de salud, lo que es esencial para hablar de integralidad en la prestación de servicios médico-asistenciales. A este respecto, la Ley Estatutaria en Salud (1751 del2015) define la atención integral en términos de prevención, paliación o curación de la enfermedad (15) y, para dar respuesta a ello, los modelos deben contener los objetivos, los principios o la fundamentación que los orienta, así como los componentes operativos para lograrlo. La revisión de estos elementos en las instituciones participantes permite decir que estas dan cuenta de la importancia de la atención integral, pero no en todas ellas queda explícita la forma de llevarla a cabo.

$\mathrm{Al}$ respecto cabe agregar que la fundamentación del modelo de atención debe plasmarse en forma expresa. Esta dependerá del enfoque: si se trata de un modelo que privilegia la integralidad se debe gestionar la salud y sus posibles determinantes; si es la gestión del riesgo, debe permitir la identificación oportuna de este, y si es la gestión de la atención clínica, debe favorecer la recuperación de la salud con criterios óptimos de calidad (16). Adicionalmente, debe especificarse el componente operativo del modelo, en el cual es fundamental definir los grupos de atención por ciclos vitales y familiares, espacios o escenarios donde se desarrolla la atención ambulatoria y de internación, así como la integración entre servicios internos y externos (17).

\section{Enfoque de los programas y las actividades}

A partir de los resultados expuestos, se puede señalar que el nivel de complejidad de las instituciones participantes demarca un enfoque de prevención terciaria en la mayoría de programas y las actividades que desarrollan, puesto que atender personas en estado de enfermedad significa el despliegue de acciones encaminadas a recuperar la salud y evitar complicaciones. No obstante, se pudo identificar que estas instituciones también contemplan programas y actividades que van más allá y se enmarcan en lo que se conoce como promoción de la salud, prevención de la enfermedad primaria y secundaria, las cuales buscan la participación activa de los 
pacientes en sus procesos de salud-enfermedad. Estos hallazgos se relacionan con lo expuesto por algunos autores cuando afirman que las acciones de promoción están someramente estipuladas en el sistema de salud colombiano, así algunas instituciones las desarrollen como valor agregado de su atención o como estrategia para disminuir costos hospitalarios (18-19).

Los programas y las actividades que se desarrollan en las instituciones participantes no están clasificados explícitamente en las categorías de promoción de la salud o prevención de la enfermedad como tal. Sin embargo, se puede ver una tendencia preventiva en la mayoría de los casos, lo que está directamente relacionado con la dinámica, las características, las necesidades y el nivel de complejidad de las instituciones estudiadas. Si bien la mayoría de las acciones tienen un enfoque preventivo, también se encontraron otras que pueden clasificare en el campo de la promoción de la salud, lo cual es relevante en la medida que el cuidado de la salud debe tener un enfoque integral (20).

Con respecto a la prevención, es necesario recordar que esta busca el control de las enfermedades y pone el énfasis en los factores y las poblaciones de riesgo. La promoción, por el contrario, está centrada en la salud misma y pone su acento en los determinantes sociales de la salud (21). La comprensión de estos significados tiene sentido puesto que permite ampliar la visión del alcance de lo que se hace y lo que se puede llegar a hacer en materia de promoción de la salud, prevención de la enfermedad, curación y rehabilitación.

En el contexto de la promoción de la salud, se encontró que en algunas de estas instituciones se realizan actividades culturales, deportivas y educativas dirigidas al personal que labora en ellas, las cuales hacen parte de las estrategias de promoción de la salud dispuestas en la Carta de Ottawa desde 1986, y buscan generar conductas saludables y la utilización de servicios creados para la garantía de sus derechos como trabajadores y ciudadanos (1). También en algunos casos la comunidad en general se beneficia de programas educativos, orientados al fomento de hábitos saludables que pueden incidir en el estado de salud de las personas, así como mejorar su acceso a los servicios de salud (22).

Como parte de la prevención primaria, se halló que para evitar riesgos potenciales, las instituciones participantes hacen uso de diferentes estrategias de información, educación y comunicación (IEC), que van desde actividades educativas colectivas hasta el uso de programas radiales, de televisión y, en general, de tecnologías de la información, todos ellos orientados a personas que hacen parte de la institución y a otros públicos de diversas características y contextos, cuyo énfasis es el fomento de prácticas que reducen riesgos específicos, orientación principal que deben tener las acciones de este nivel de prevención (21).

En cuanto a la prevención secundaria, las actividades que se realizan están orientadas a dotar a los pacientes y a sus familias o cuidadores principales, de información valiosa para que puedan participar activamente de su tratamiento. La mayoría de las instituciones identifican desde el ingreso, las necesidades educativas manifiestas y potenciales de los pacientes y sus cuidadores. De acuerdo a estas, se planean acciones que se desarrollan durante la estancia hospitalaria y, en algunos casos, posterior a esta. Asimismo, los pacientes con enfermedades crónicas de alta incidencia participan de programas que les facilitan el manejo y control de su patología, y encuentran, además de apoyo a su desequilibrio biológico, alternativas para cuidar su salud mental y social. De 
esta manera, se cumple con los objetivos de la prevención secundaria, al tratar de hacer diagnósticos tempranos, captación oportuna y tratamiento adecuado, esenciales para el control de la enfermedad (21).

La prevención terciaria se hace protagonista, en la medida que el perfil de morbimortalidad de nuestro contexto refleja un aumento significativo de algunas enfermedades crónicas que requieren no solo de tratamientos prolongados y seguimiento constante, sino también cambios de los comportamientos en la dinámica personal y familiar de quien las sufre. De esta manera, las instituciones participantes han diseñado estrategias para brindar cuidado integral a sus pacientes, por medio de encuentros educativos, entrenamientos y controles especializados que dan valor agregado a sus servicios, involucrando tanto al paciente como a su familia o cuidador principal. Lo anterior es congruente con las acciones de control y seguimiento propias de la prevención terciaria, las cuales buscan, entre otros objetivos, facilitar la aplicación de tratamientos y medidas de rehabilitación, minimizar los sufrimientos causados al perder la salud, facilitar la adaptación de los pacientes a problemas incurables y contribuir a prevenir o reducir al máximo las recidivas de la enfermedad (23).

A partir de lo anterior, se puede concluir que, si bien en las instituciones priman los programas y las actividades de prevención terciaria afines al nivel de complejidad de estas, también se desarrollan acciones orientadas a la promoción de la salud y la prevención primaria y secundaria de la enfermedad. No obstante, es importante aclarar que la diferenciación del enfoque entre los diferentes niveles fue complejo, tanto para las instituciones de salud como para los investigadores, en la medida que los límites entre ellos no son tan evidentes y que sus acciones se entretejen.

\section{Públicos beneficiarios}

Otro elemento importante que considerar en la presente discusión tiene que ver con los beneficiarios de los programas y las actividades de promoción o prevención, es decir, el público objetivo. Las acciones de este tipo desarrolladas en las instituciones del estudio están dirigidas, principalmente, a los pacientes y sus familias, lo cual es válido en la medida que un hospital de alta complejidad está centrado, en particular, en la atención del individuo enfermo y por ello canaliza sus esfuerzos curativos y preventivos a este nivel. Empero, debe anotarse que se han identificado otros beneficiarios que deben mencionarse, por su importancia en el contexto de la promoción de la salud y la prevención de la enfermedad. Es el caso del personal de la institución y la comunidad.

Lo anterior nos remite, necesariamente, a la propuesta de Hospitales Promotores de la Salud, la cual considera estrategias orientadas a los pacientes, el personal (staff) y la comunidad donde se sitúa la institución. Se busca fortalecer en todos estos públicos el empoderamiento en materia de autocuidado y el fomento de estilos de vida saludables (24).

De acuerdo con lo mencionado, el grupo de pacientes es el público objetivo por excelencia en el ámbito hospitalario. Los resultados señalan que las intervenciones informativas, comunicativas y educativas que se dirigen a los pacientes y familias buscan, sobre todo, aportar elementos para el fomento del autocuidado en el contexto hospitalario y, posteriormente, en el domicilio. Estos resultados pueden quedarse cortos si tenemos en cuenta las posibilidades existentes en materia de estrategias en los Hospitales Promotores de la Salud, las cuales se centran, entre otros aspectos, en lo siguiente: fomento del autocuidado en el hospital; capacitación de los pacientes para afianzar su participación en su 
tratamiento y cuidado; empoderamiento de pacientes para la gestión de las enfermedades crónicas y en la adquisición de estilos de vida saludables; mejoramiento del entorno hospitalario en pro de los pacientes y de las condiciones de la infraestructura comunitaria, relacionadas con las necesidades específicas de estos (24).

El personal del hospital es otro de los públicos considerados por las instituciones estudiadas, en su labor de promocionar la salud y prevenir la enfermedad, la cual se desarrolla, primordialmente, a través de los programas de salud ocupacional que por ley deben existir $\mathrm{y}$, en algunas ocasiones, mediante programas complementarios de bienestar general que tienen algunas de ellas, los cuales deben ser reconocidos por su importancia para el cuidado de la salud de los trabajadores. La inclusión de este público es clave dentro del contexto de los Hospitales Promotores de la Salud y debe hacerse mediante el desarrollo de estrategias diversas, algunas de ellas orientadas al personal directamente y otras centradas en el hospital como lugar de trabajo, las cuales buscan que las personas se empoderen de su cuidado, sean capaces de autocontrolar los determinantes de la salud relacionados con el trabajo y estén facultadas para adoptar estilos de vida saludables. Adicionalmente, las estrategias se orientan a desarrollar en los hospitales y fuera de ellos, las condiciones de infraestructura necesarias para promover la salud y mejorar las condiciones de vida del personal (24).

La comunidad también se beneficia de los programas y las actividades desarrollados desde las instituciones consultadas. Se identificaron tres tipos de beneficiarios en este contexto. Uno de ellos está constituido por grupos de personas captadas en función de los riesgos específicos que presentan, y no por la ubicación geográfica o área de referencia hospitalaria. Otro tipo de beneficiarios lo constituyen franjas de población general que tienen acceso a las acciones educativas que desarrollan las instituciones a escala extramural. Y, de manera puntual en una de las instituciones, el público corresponde a una comunidad específica donde se llevan a cabo acciones diversas de empoderamiento de líderes y desarrollo de capacidades en el personal del hospital de esta población.

Amén de las actividades que desarrollan las instituciones participantes, la estrategia de Hospitales Promotores de la Salud propone otro tipo de acciones que es importante considerar, tales como: promover el acceso de la población a los servicios hospitalarios; fortalecer la capacidad de los cuidadores o personal del nivel primario para el manejo de los pacientes luego del alta; procurar que el hospital como centro tenga las puertas abiertas al desarrollo de otras actividades promotoras de la salud (culturales, deportivas, etc.); empoderar a la población en la gestión de la enfermedad crónica y en la adopción de estilos de vida saludables; $y$, en último término, ayudar a que la comunidad como un todo mejore sus condiciones generales y promueva así su desarrollo. Lo anterior contribuye también al logro de los objetivos relacionados con la responsabilidad social corporativa, elemento que se destaca en el modelo (25).

Lo encontrado muestra que las instituciones desarrollan programas y actividades de promoción y prevención a grupos diversos —paciente, familia, personal y comunidad-, lo que permite ver una aproximación con respecto a los lineamientos de los Hospitales Promotores de la Salud. No obstante, este modelo considera una gama más amplia de estrategias que pueden servir de referente para los hospitales de alta complejidad a la hora de proyectarse como una institución que brinda atención integral en materia de promoción, prevención, atención y rehabilitación. 


\section{Equipos de trabajo}

Otro de los puntos que destacar tiene que ver con las características de los equipos de trabajo que desarrollan actividades de promoción de la salud y prevención de la enfermedad en las instituciones, lo cual es esencial a la hora de abordar de manera integral el proceso salud-enfermedad donde las diferentes disciplinas tienen algo que aportar.

Lo anterior nos remite al tema del trabajo interdisciplinario de los actores implicados, que, si bien no se estudió a profundidad en este caso, sí se alcanzó a observar durante el proceso investigativo que el trabajo en las instituciones tiene un carácter más multidisciplinario que interdisciplinario, dado que se enfoca en la suma de actividades de diferentes profesionales alrededor de una temática específica. Por esto, es necesario que las instituciones cuenten con equipos que, de manera "intencional, explícita, controlable y selectiva”, aborden el trabajo a favor de la promoción y la prevención en forma cooperativa, porque la interdisciplinariedad se caracteriza, precisamente, por eso, la integración en red de un grupo de profesionales quienes comparten marcos conceptuales y metodológicos para construir un saber común alrededor de un problema que plantean conjuntamente $(26,27)$.

Este resultado también se ha identificado en otros trabajos desarrollados en el campo de la promoción de la salud y la prevención de la enfermedad; por ejemplo, el realizado por Ríos et al. (28), en el cual se estudió la experiencia de un grupo de bacteriólogos que se desempeñaban en este ámbito. Allí se concluyó que, "a pesar de tener clara la necesidad de requerirse las múltiples miradas y de ubicar la interdisciplinariedad y el trabajo en equipo como los pilares para el trabajo en promoción y prevención, se vio que en la práctica el trabajo se segmenta, cada uno de los profesionales realiza únicamente las tareas que le competen a su profesión y no se crea una visión compartida”.

El análisis de las distintas experiencias estudiadas también permitió identificar grupos de trabajo que han tenido avances en su forma de abordar las actividades de promoción y prevención, principalmente la labor educativa. En estos grupos el poder se ha descentrado del médico y existe una participación activa de otros profesionales, entre ellos el psicólogo, el profesional de enfermería, el comunicador o el trabajador social, entre otros. Además, se hace un abordaje más integral de la persona, considerando factores psicológicos, culturales y sociales que protegen o limitan los procesos de promoción y prevención. Es el caso de una de las instituciones donde el abordaje se realiza desde la perspectiva psicoeducativa.

Los resultados referidos a los equipos de trabajo dan cuenta también de la participación de los profesionales del área de comunicaciones en el desarrollo de programas y actividades de promoción y prevención. Esta participación es trasversal a todos los procesos de información, educación y comunicación que se desarrollan, en algunos casos como apoyo a los diferentes programas y actividades; en otros, los profesionales del área de comunicación son directamente responsable de su ejecución.

\section{Metodologías o estrategias educativas}

Según los hallazgos, el tema de las metodologías y estrategias educativas hace alusión a la diversidad de herramientas usadas en las instituciones participantes, para el desarrollo de actividades que tienen como fin fomentar la salud, prevenir enfermedades o complicaciones, orientadas a sus diferentes actores, principalmente al paciente y su familia. 
En la mayoría de los casos, los programas y las actividades que se desarrollan en estas instituciones para promover la salud o prevenir la enfermedad tienen un enfoque educativo, tanto a escala individual y familiar como a escala colectiva, y hacen uso de metodologías directas e indirectas. Las primeras se realizan a través de encuentros entre profesionales de la salud y una persona o grupo de personas. Las indirectas, por su parte, se desarrollan principalmente por medio de tecnologías de información y comunicación (TIC).

Los encuentros, como metodologías educativas directas, se han definido como un espacio donde la comunicación, la comprensión y el respeto por el otro son los pilares del aprendizaje. Allí la persona cuenta con el apoyo profesional requerido; sin embargo, la responsabilidad del cuidado de sí, recae en ella. De esta manera, es mucho más factible que alcance el empoderamiento necesario para su autocuidado (29). Estos encuentros, además, deben estar mediados por la interacción dialógica, la cual se basa en una comunicación que lleva a todos los sujetos implicados a compartir una acción, acuerdo, sentimiento o deseo, lo que da sentido a las acciones educativas que buscan cambios de comportamiento para mejorar la salud (30).

Los encuentros educativos dirigidos a los pacientes y sus familias buscan que estos comprendan su proceso salud-enfermedad y asuman un rol relevante en la gestión de sus riesgos, tratamiento y rehabilitación. E1 entrenamiento sobre cuidados básicos también hace parte de este tipo de estrategias e involucra a la familia o cuidador principal como actor fundamental del proceso de prevención (31).

Para el desarrollo de actividades educativas directas de promoción y prevención dirigidas a colectivos, las instituciones realizan jornadas o ferias de salud, talleres y conferencias, en las cuales participan grupos interdisciplinarios, e incluso interinstitucionales, de acuerdo con el alcance de la actividad o programa. Una de las posibles amenazas que pueden llegar a tener los encuentros colectivos es que muchas veces se desarrollan de forma magistral, dirigidos a públicos con necesidades muy diversas, que no permiten atender las necesidades de aprendizaje particulares, ni la interacción entre las personas. En este sentido, autores que han trabajado la educación para la salud en el ámbito colectivo, recomiendan que se lleven a cabo encuentros con públicos pequeños, con características sociodemográficas, clínicas o necesidades similares, para que se puedan tejer relaciones basadas en las experiencias, los aciertos y los desaciertos en el control de sus enfermedades o riesgos, y que esto sirva a todos para comprender mejor su papel protagónico en la conservación de la salud (32).

Las TIC, tales como páginas web, videos, internet, sistemas internos y externos de televisión, redes sociales, teléfono y programas radiales, son algunas de las herramientas metodológicas indirectas utilizadas para el desarrollo de actividades educativas, lo que podría entenderse como parte de la incorporación del desarrollo tecnológico al sector salud y la expansión del acceso de información a las personas. $\mathrm{Al}$ respecto, es importante mencionar que su utilización efectiva depende, en gran medida, del entrenamiento que se tenga para aprovechar al máximo las oportunidades que ofrecen. Así lo refiere D' Agostino, cuando asegura que el personal de salud debe ser alfabetizado para la incorporación de nuevas tecnologías de información y que de esto dependerá que sus repercusiones sean positivas o perjudiciales para los pacientes (33).

El material impreso se identificó como una ayuda educativa representativa en las instituciones del estudio. Su uso es importante, en la 
medida que sirve de apoyo tanto al profesional como al paciente para consolidar los aspectos claves de la educación recibida y favorecer el acceso rápido a información precisa y de utilidad relacionada con un estado particular de salud. Es importante mencionar que este tipo de material educativo debe obedecer a un proceso riguroso de elaboración. De lo contrario, su propósito educativo se puede desvirtuar. Este tipo de ayudas por sí solas no tienen mayor valor, este radica en ser instrumentos que facilitan el aprendizaje y deben ser parte de una serie de estrategias implementadas para el logro de un objetivo educativo definido (34).

\section{Conclusión}

A partir del análisis realizado, se puede concluir que las instituciones participantes desarrollan actividades de promoción de la salud y prevención de la enfermedad, con un enfoque hacia la prevención terciaria congruente con su nivel de complejidad, y tienen un énfasis educativo que se desarrolla a través de diferentes medios de comunicación. Asimismo, se orientan al paciente, principalmente, con participación de las familias, y, en algunos casos, a la comunidad. Además, se benefician los empleados, desde la mirada de salud ocupacional. Estas actividades son desarrolladas por profesionales de diferentes disciplinas y su estructura está ligada, en muchos de los casos, a las direcciones científicas, con excepción de las áreas de comunicación y salud ocupacional. No se identifica un área en particular que las gestione de forma articulada para hacerlas más representativas en el modelo de atención integral de cada institución.

Las instituciones de salud deben fortalecer sus modelos de atención en salud, permitiendo una mayor articulación de las actividades de promoción de la salud y prevención de la enfermedad con la atención clínica, utilizando modelos como el planteado por la OMS, Hospital Promotor de la Salud, y dinámicas de clínicas preventivas que se enfoquen en el cuidado integral de toda la población.

\section{Referencias bibliográficas}

1. Conferencia Internacional de Promoción de la Salud. Carta de Ottawa: Salud para todos en el año 2000. Ottawa, 1986 [acceso: 12 de abril del 2013]. Disponible en: http://www.paho.org/spanish/hpp/ ottawachartersp.pdf

2. Nutbeam D. Health promotion glossary. Health Promotion International. 1998;13 [acceso: 17 de marzo del 2017]. Disponible en: https://academic. oup.com/heapro/article/15/3/259/551108/Health-literacy-as-a-public-health-goal-a\#8715851

3. Leavell HA, Clark EG. Preventive medicine for the doctor in his community: an epi-demiologic approach. Nueva York: McGraw-Hill; 1965.

4. Molina de Salazar DI. Propuesta en prevención del riesgo cardiovascular. Rev Colomb Cardiol. 2008;15(5):203-6.

5. Eslava JC. Promoción y prevención en el sistema de salud en Colombia. Rev Salud Pública. 2002;4(1):112.

6. Ruales J. Panel: tendencias en servicios de salud y modelos de atención de la salud. Rev Fac Nac Salud Pública. 2004;22(99):33-9.

7. Giraldo Osorio A, Toro Rosero MY, Macías Ladino AM, Valencia Garcés CA, Palacio Rodríguez S. La promoción de la salud como estrategia para el fomento de estilos de vida saludables. Rev Hacia Promoc Salud. 2010;15(1):128-43.

8. Rosengren A, Hawken S, Ôunpuu S, Sliwa K, Zubaid M, Almahmeed WA, et al. Association of psychosocial risk factors with risk of acute myocardial infarction in 11119 cases and 13648 controls from 52 countries (the INTERHEART study): case-control study. Lancet. 2004;364(9438):953-62.

9. Johnson A, Baum F. Health promoting hospitals: a typology of different organizational approaches to health promotion. Health Promot Int. 2001;16(3):281-7.

10. García G, Rodríguez M, Benia W. Niveles y estrategias de prevención. En: Benia W. Temas de Salud Pública, tomo I. Montevideo: Fefmur; 2008. p. 19-26.

11. Decreto 1876 de 1994. Diario Oficial, n. ${ }^{\circ} 41.480$ (5 de agosto de 1994).

12. Delgado A. Evaluación de la calidad de la atención en salud, un primer paso para la reforma del sistema. Salud Uninorte. 2010;26(1):143-54.

13. Resolución 00412 de 2000 . Ministerio de Salud, 25 de febrero del 2000, Bogotá.

14. Infante A, De la Mata I, López-Acuña D. Reforma de los sistemas de salud en América Latina y el Caribe: situación y tendencias. Pan Am J Public Health. 2000; 8:13-20. 
15. Ley 1751 de 2015, Diario Oficial, n. ${ }^{\circ} 49427,16$ de febrero del 2015.

16. Grosso Sandoval G, Gómez Rivadeneira A, Fernando Betancourt V. Modelos de salud: administración de planes de beneficios desde la perspectiva de la gestión del riesgo. Bogotá: Universidad Nacional de Colombia; 2006.

17. Ramírez NA, Romero RV. El caso de la implementación de la estrategia de Atención Primaria Integral en Salud (APIS) en la localidad de Suba, Bogotá-Colombia. Rev Gerenc Políticas Salud. 2008; 7(14):125-144.

18. Villamil NR, González AV, Mosquera PD. Promoción de la salud y sistema de salud colombiano: Reflexiones sobre la formación de profesionales. Rev Hacia Promoc Salud. 2013;18(1):26-40.

19. Villarreal-Ríos E, Mathew-Quiroz A, Garza-Elizondo ME, Núñez-Rocha G, Salinas-Martínez AM, Gallegos-Handal M. Costo de la atención de la hipertensión arterial y su impacto en el presupuesto destinado a la salud en México. Salud Pública Mex. 2002;44(1):7-13.

20. Franco-Giraldo Á. La última reforma del sistema general de seguridad social en salud colombiano. Rev Salud Pública. 2012;14(5):865-77.

21. Vignolo J, Vacarezza M, Álvarez C, Sosa A. Niveles de atención, de prevención y atención primaria de la salud. Arch Med Interna. 2011;33(1):7-11.

22. Organización Mundial de la Salud (OMS). Cuarta Conferencia Internacional sobre Promoción de la Salud: nueva era, nuevos actores: adaptar la Promoción de la Salud al siglo XXI. Declaración de Yakarta. Yakarta, Java: Organización Mundial de la Salud; 1997.

23. Vignolo J, Vacarezza M, Álvarez C, Sosa A. Niveles de atención, de prevención y atención primaria de la salud. Arch Med Int [Internet]. 2011 Abr [acceso: 17 de marzo del 2017]; 33(1):7-11. Disponible en: http://www.scielo.edu.uy/scielo.php?script $=$ sci_arttext\&pid=S1688-423X2011000100003\&lng =es

24. Groene O, Garcia-Barbero M. Health promotion in hospitals: evidence and quality management [internet]. Copenhagen, Denmark: WHO; 2005 [acceso: 11 de mayo del 2016]. Disponible en: https://www.researchgate.net/profile/Oliver_Groene/ publication/240633752 Health Promotion in Hospitals_Evidence_and_Quality_Management/ links/004635356465505121000000.pdf

25. Garcia-Barbero M. The Health Promoting Hospitals Movement. Journal of European Private Hospitals. J Eur Priv Hosp. 1994:33-6.

26. Schejter V, Selvatici L, Cegatti J, De Raco PP, Ugo F, Jesiotr M. Imaginarizar el trabajo interdisciplinario en salud. Anu Investig. 2006;13:195-200.

27. Allegro F, Beltrán F, Benmergui S, Molina JL, Oszlak C, Panelo A. Propuesta para la formación de equipo interdisciplinario en Salud: facilidades e inconvenientes. Boletín Científico. 2007;12(56):1-3.

28. Ríos M, Duarte D, Mesa R, Zapata B, Palacio S. Nociones y experiencias en promoción de la salud y prevención de la enfermedad de bacteriólogos y laboratoristas clínicos egresados de la Universidad de Antioquia. Rev Fac Nac Salud Pública. 2005;23(1):19-31.

29. Molina DR. La salud pública como ciencia social: reflexiones en torno a las posibilidades de una salud pública comprensiva. Rev Fac Nac Salud Pública. 2011;29(1):95-102.

30. Soler M, Flecha R. Desde los actos de habla de Austin a los actos comunicativos: Perspectivas desde Searle, Habermas y CREA. Rev Signos. 2010;43:363-75.

31. Barrera Ortiz L, Galvis López CR, Moreno Fergusson ME, Pinto Afanador N, Pinzón Rocha ML, Romero González E, et al. La habilidad de cuidado de los cuidadores familiares de personas con enfermedad crónica: un estudio comparativo de tres unidades académicas de enfermería. Investig Educ En Enferm. 2006;24(1):36-46.

32. Ledón Llanes L. La comunicación en el campo de la diabetes mellitus, algunas pautas para su acción efectiva. Rev Cuba Endocrinol. 2014; 25(2):124-38.

33. D' Agostino M. Estrategias de salud electrónica en la región de las Américas: situación actual y perspectivas. Rev Peru Med Exp Salud Pública. 2015;32(2):352-5.

34. Gallego Diéguez J. Materiales educativos en educación para la salud. Criterios de análisis y elaboración. Aragón, España: Dirección General de Salud Pública; 2008. 